\title{
Research on Stability Control Based on the Wheel Speed Difference for the AT Vehicles
}

\author{
Hui Jin and Shijie Li \\ School of Mechanical Engineering, Beijing Institute of Technology, Beijing 100081, China \\ Correspondence should be addressed to Hui Jin; jinhui@bit.edu.cn
}

Received 12 July 2014; Accepted 25 August 2014

Academic Editor: Heiner Bubb

Copyright (C) 2015 H. Jin and S. Li. This is an open access article distributed under the Creative Commons Attribution License, which permits unrestricted use, distribution, and reproduction in any medium, provided the original work is properly cited.

This paper utilizes a linear two-degree-of-freedom vehicle model to calculate the nominal value of the vehicle's nondrive-wheel speed difference and investigates methods of estimating the yaw acceleration and sideslip angular speed. A vehicular dynamic stability control system utilizing this nondrive-wheel speed difference is then developed, which can effectively improve a vehicle's dynamic stability at a very low cost. Vehicle cornering processes on roads of different frictions and with different vehicle speeds are explored via simulation, with speed control being applied when vehicle speed is high enough to make the vehicle unstable. Driving simulator tests of vehicle cornering capacity on roads of different friction coefficients are also conducted.

\section{Introduction}

When vehicles turn on low friction roads or steer while travelling at a high speed, if the lateral force provided by the tires is close to their adhesion limit, then the car will enter a dangerous state of operation, with increased risk of sideslip, sharper turning, or reduced responsiveness. Dynamic stability control systems can significantly improve vehicle cornering performance, and so they have become the focus of intensive research and development in recent years [1-7]. The researches are mainly concentrated in two aspects. One is on the vehicle simulation model establishment, either with Matlab/Simulink [8] or with Simulink and ADAMS [9]. The other is on the stability control using different control strategies [10]. Use of such systems has however been limited by their high cost. For cars already equipped with automatic transmission, however, dynamic stability control can actually be realized simply by adding two nondrive-wheel speed sensors to the existing system. Such a system is very cheap and has proven to be capable of greatly improving the active safety of AT cars on crooked roads: it hence has great potential for the application in compact cars. This paper thus develops a vehicular dynamic stability control system based on the nondrive-wheel speed difference.

The core concept of vehicular dynamics control is preventing vehicles from entering an unsteady stage by enhancing control during the quasi-steady stage. When the cornering characteristics of the vehicle's tires enter the nonlinear zone, the lateral force imposed on the tires fails to maintain a linear relationship with the sideslip angle of the tires, leading to a large difference between the actual dynamic characteristics of the vehicle and those calculated according to a linear vehicle model. The physical properties of a vehicle's movement, such as its lateral acceleration, yaw velocity, and sideslip angle, can be used to describe its lateral dynamic characteristics, and the measured values of these quantities are what deviate from their theoretically predicted values when the cornering characteristics of a tire enter the nonlinear zone. The stability of vehicles can thus be deduced by analyzing the difference between the measured values and the nominal values of the abovementioned physical quantities. A vehicle's cornering condition can be recognized as in a steady stage if said difference is small, while if it exceeds a preset range then the vehicle may be considered to have entered a quasi-steady stage, and dynamic stability control becomes necessary [11-20]. Measurement of properties such as lateral acceleration, yaw velocity, and sideslip angle requires special sensors that are generally expensive. To reduce this cost as much as possible, this paper hence utilizes the speed difference of nondrive wheels to judge the dynamic stability of a vehicle, on the following grounds: (1) the speed difference of nondrive wheels is directly proportional to the yaw velocity; 


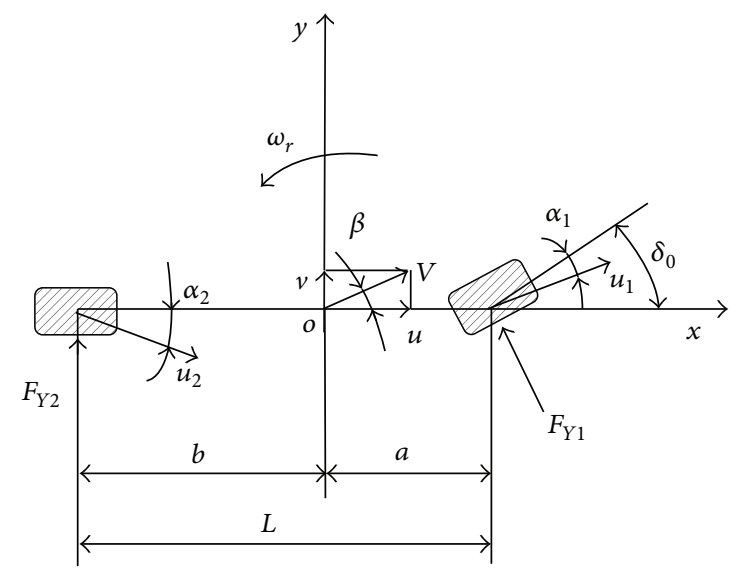

FIGURE 1: 2-DOF vehicle model.

(2) the inner and outer speed of nondrive wheels can easily be measured by installing speed sensors, which are quite cheap.

\section{Calculation of Nominal Value of Wheel Speed Difference}

The following assumptions are made during construction of the linear 2-degree-of-freedom model: (1) influence from the steering system is negligible and the front-wheel cornering angle is input directly; (2) the function of the suspension is negligible and the car only moves in a plane parallel to the ground; that is, displacement along the $z$-axis, the pitch angle from the $y$-axis, and the roll angle from the $x$-axis are zero; (3) the forward speed of the car along the $x$-axis is constant. Based on such simplifications, the vehicle only has two degrees of freedom: lateral movement along the $y$-axis and yaw movement along the $z$-axis. The linear two-degreeof-freedom vehicle model is shown in Figure 1 and the $z$-axis is perpendicular to the ground plane.

The differential equation for vehicle motion in 2-degreeof-freedom can be established as

$$
\begin{aligned}
& \left(k_{1}+k_{2}\right) \beta+\frac{1}{u}\left(a k_{1}-b k_{2}\right) \omega_{r}-k_{1} \delta_{0}=m\left(\dot{v}+u \omega_{r}\right), \\
& \left(a k_{1}-b k_{2}\right) \beta+\frac{1}{u}\left(a^{2} k_{1}+b^{2} k_{2}\right) \omega_{r}-a k_{1} \delta_{0}=I_{z} \dot{\omega}_{r} .
\end{aligned}
$$

The meaning of the symbols in Figure 1 and Formula (1) is listed in Table 1.

The equation $\beta=v / u$ can be written as $v=\beta \cdot u$, by derivation on both sides:

$$
\dot{v}=\dot{\beta} \cdot u
$$

By substituting Formula (2) into Formula (1), we obtain

$$
\begin{aligned}
& m u\left(\dot{\beta}+\omega_{r}\right)=\left(k_{1}+k_{2}\right) \beta+\frac{1}{u}\left(a k_{1}-b k_{2}\right) \omega_{r}-k_{1} \delta_{0}, \\
& I_{z} \dot{\omega}_{r}=\left(a k_{1}-b k_{2}\right) \beta+\frac{1}{u}\left(a^{2} k_{1}+b^{2} k_{2}\right) \omega_{r}-a k_{1} \delta_{0} .
\end{aligned}
$$

TABLE 1: Implication of all symbols.

\begin{tabular}{ll}
\hline Symbols & Implication \\
\hline$k_{1}$ & Front-wheel cornering stiffness \\
$k_{2}$ & Rear-wheel cornering stiffness \\
$\beta$ & Sideslip angle of center of mass \\
$u$ & Longitudinal velocity of center of mass \\
$v$ & Lateral velocity of center of mass \\
$a$ & Distance from front axle to center of mass \\
$b$ & Distance from rear axle to center of mass \\
$L$ & Wheel space \\
$u_{1}$ & Velocity of front axle center \\
$u_{2}$ & Velocity of rear axle center \\
$\omega_{r}$ & Yaw velocity \\
$\delta_{0}$ & Front-wheel cornering angle \\
$m$ & Vehicle mass \\
$\dot{v}$ & Lateral acceleration of center of mass \\
$I_{z}$ & Moment of inertia along coordinate $z$ \\
$\dot{\omega}_{r}$ & Yaw acceleration \\
$\alpha_{1}$ & Front-wheel sideslip angle \\
$\alpha_{2}$ & Rear-wheel sideslip angle \\
$F_{Y 1}$ & Lateral force of ground to front wheels \\
$F_{Y 2}$ & Lateral force of ground to rear wheels \\
\hline
\end{tabular}

The above formula can be solved with $\beta$ and $\omega_{r}$ as variables:

$$
\begin{aligned}
& \omega_{r} \\
& =\frac{u\left[\delta_{0} \cdot k_{1} \cdot k_{2} \cdot L+I_{z} \dot{\omega}_{r}\left(k_{1}+k_{2}\right)-m \dot{\beta} u\left(a k_{1}-b k_{2}\right)\right]}{k_{1} \cdot k_{2} \cdot L^{2}+m u^{2}\left(a k_{1}-b k_{2}\right)} .
\end{aligned}
$$

The nominal value of the wheel speed difference is thus

$$
\begin{aligned}
& \Delta V_{N} \\
& =B \cdot \omega_{r} \\
& =\frac{B \cdot u \cdot\left[\delta_{0} \cdot k_{1} \cdot k_{2} \cdot L+I_{z} \dot{\omega}_{r}\left(k_{1}+k_{2}\right)-m \dot{\beta} u\left(a k_{1}-b k_{2}\right)\right]}{k_{1} \cdot k_{2} \cdot L^{2}+m u^{2}\left(a k_{1}-b k_{2}\right)} .
\end{aligned}
$$

In Formula (5), $B$ stands for the wheelbase of nondrive wheels, while $u$ and $\delta_{0}$ are values measured by the sensors; all values except for $\dot{\omega}_{r}$ and $\dot{\beta}$ are vehicle parameters. Since exact measurement of the yaw acceleration $\dot{\omega}_{r}$ and sideslip angular velocity $\dot{\beta}$ is difficult and requires special sensors, estimated values will be used instead. 


\section{Estimation of Yaw Acceleration $\dot{\omega}_{r}$, Sideslip Angular Speed $\dot{\beta}$, and Sideslip Angle $\beta$}

Based on the linear 2-degree-of-freedom vehicle model, we obtain

$$
\dot{\omega}_{r}=\frac{\left(a k_{1} \alpha_{1}-b k_{2} \alpha_{2}\right)}{I_{z}}
$$

Since

$$
\begin{aligned}
\alpha_{1} & =\beta+\frac{a \cdot \omega_{r}}{u}-\delta_{0}, \\
\alpha_{2} & =\beta-\frac{b \cdot \omega_{r}}{u}, \\
\beta & =\frac{v}{u}, \\
\dot{v} & =\frac{1}{m} \sum F_{y}-u \cdot \omega_{r} \\
F_{y} & =k_{1} \cdot \alpha_{1}+k_{2} \cdot \alpha_{2} .
\end{aligned}
$$

Therefore,

$$
\dot{\beta}=\frac{\dot{v}}{u}
$$

Since

$$
v=\int\left(\frac{1}{m} \sum F_{y}-u \cdot \omega_{r}\right) d t
$$

we can thus obtain $\beta=v / u$.

\section{Turning Stability Control Strategy Based on Wheel Speed Difference}

Figures 2(b) and 2(c) show the speed differences obtained through simulation of the actual tire model and linear tire model when a vehicle is moving at speeds of $14.4 \mathrm{~km} / \mathrm{h}$ and $28.8 \mathrm{~km} / \mathrm{h}$, respectively, on a road with a peak adhesion coefficient of 0.2 , where the front-wheel cornering angle complies with Figure 2(a).

We can see from Figure 2 that when the vehicle corners at a speed of $14.4 \mathrm{~km} / \mathrm{h}$, its tires work in the linear zone and the wheel speed difference calculated according to the linear model essentially coincides with the wheel speed difference calculated according to the actual tire model. This consistency between the actual value and the nominal value allows for steady driving conditions. When the vehicle corners at a speed of $28.8 \mathrm{~km} / \mathrm{h}$, however, the tires are working in the nonlinear zone and the wheel speed difference calculated according to the linear model hence differs significantly from that calculated according to the actual tire model, resulting in unsteady driving conditions.

Since the difference between the actual value and nominal value of the wheel speed difference reflects the cornering stability of the car, the dynamic control is becoming necessary to ensure stability if this difference exceeds a certain limit (difference between the actual value and the nominal value

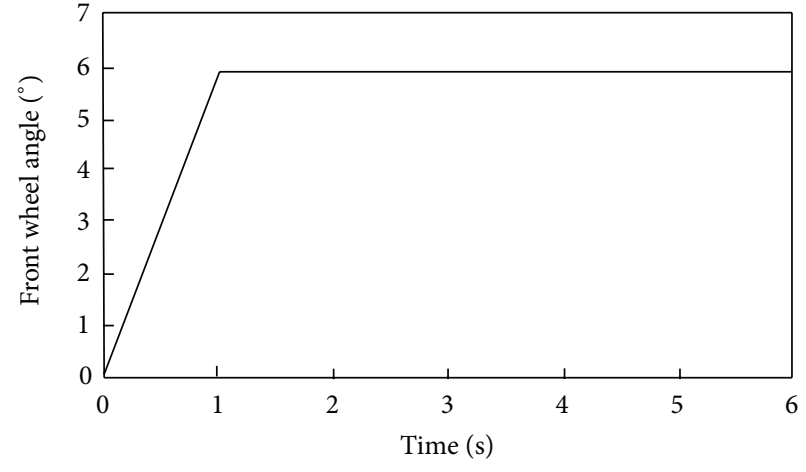

(a) Front-wheel angle input

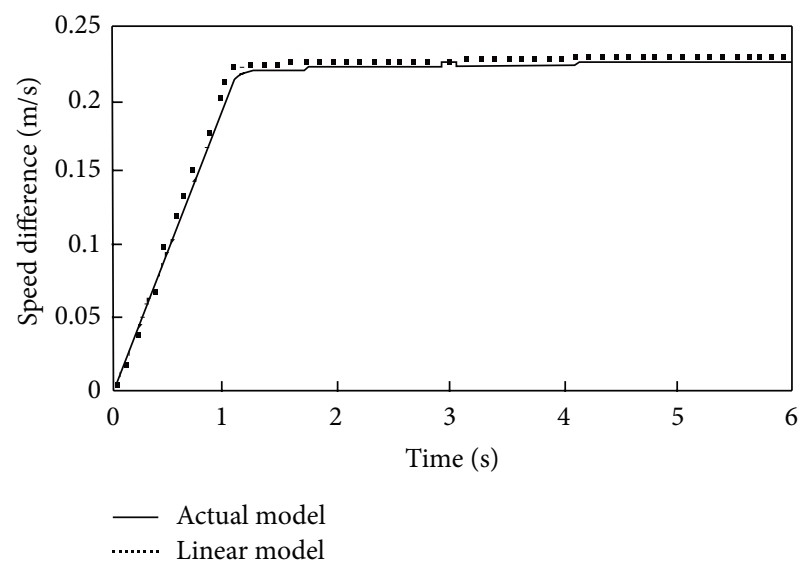

(b) Speed difference between actual model and linear model at steady stage

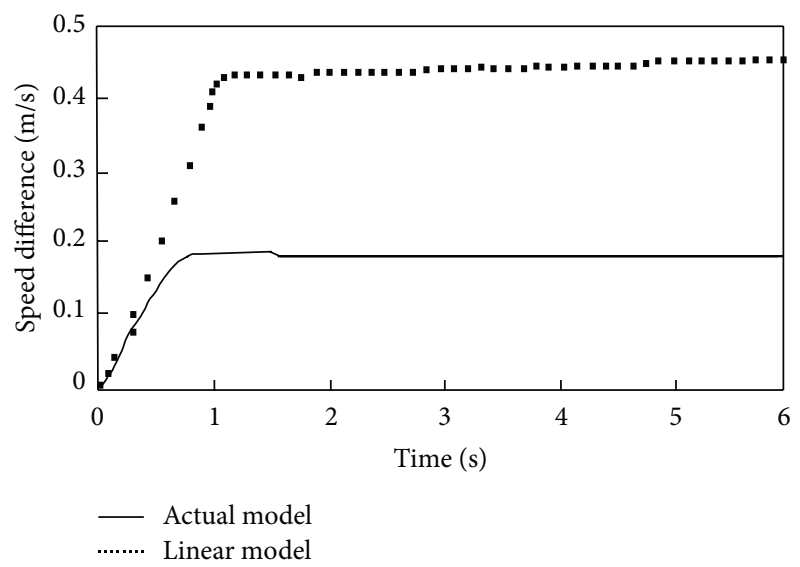

(c) Speed difference between actual model and linear model at unsteady stage

FIGURE 2: Speed difference between actual model and linear model.

is inevitable, so an upper limit and a lower limit should be defined during design of the controller). A simple and practical PI algorithm is adopted here. Control input is the speed difference between the actual value and the nominal value, and the output value is the throttle opening. Consider

$$
\theta_{\text {control }}=\theta_{\text {input }}+K_{p} \cdot e+\frac{K_{p}}{T} \cdot \int e d t
$$




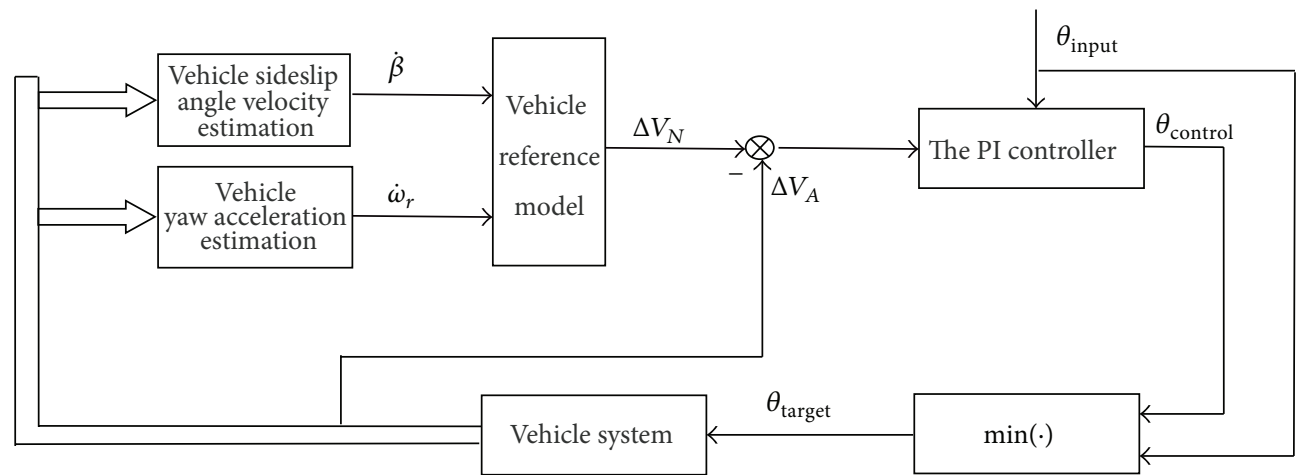

FIGURE 3: Principle of vehicle dynamic stability control chart.

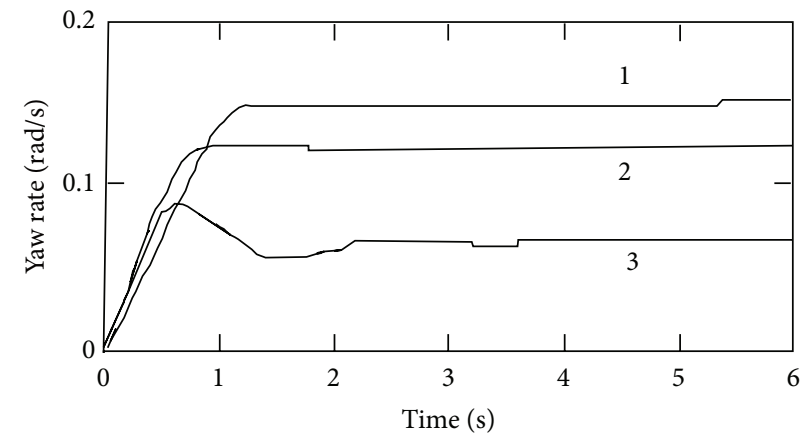

(a) The change of the yaw angular velocity

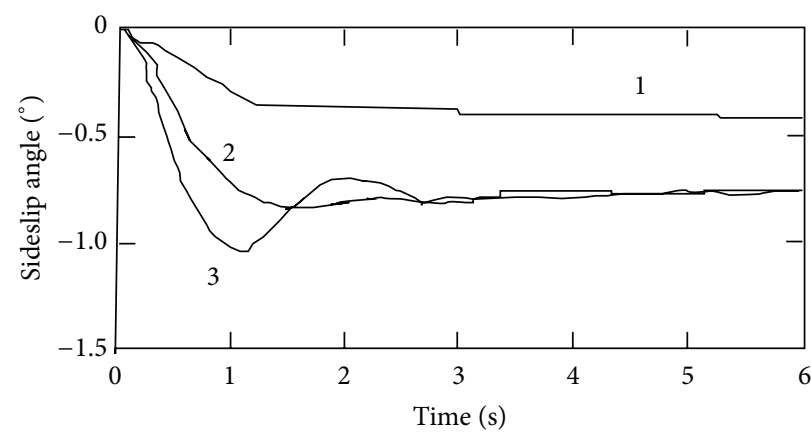

(c) The change of the rear-wheel sideslip angles

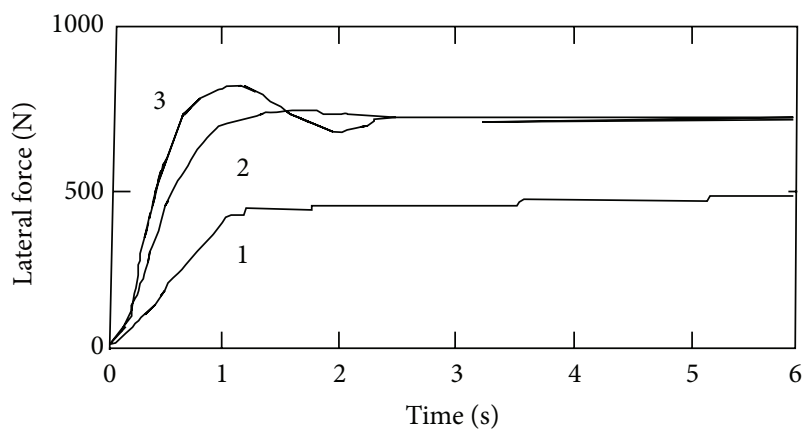

(e) Lateral force variation of the rear wheel

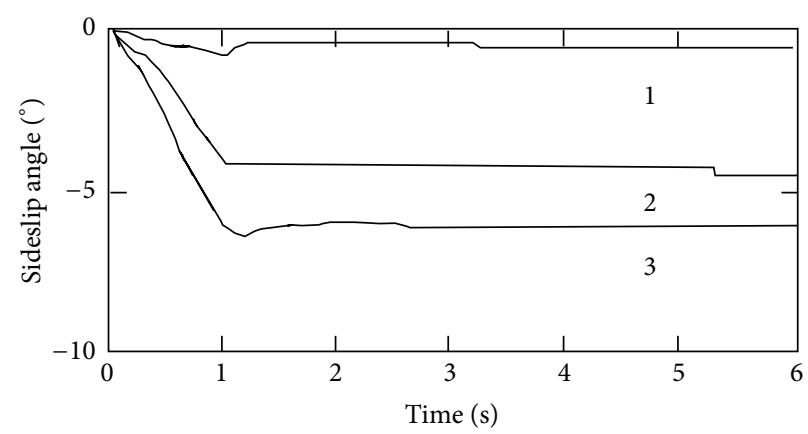

(b) The change of the front-wheel sideslip angles

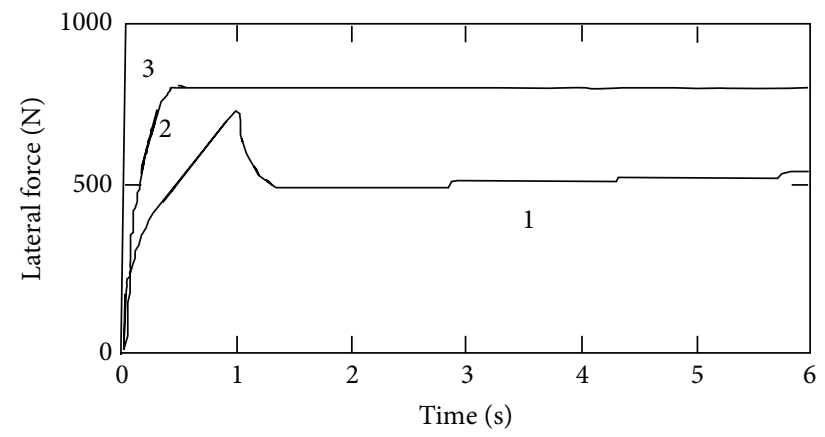

(d) Lateral force variation of the front wheel

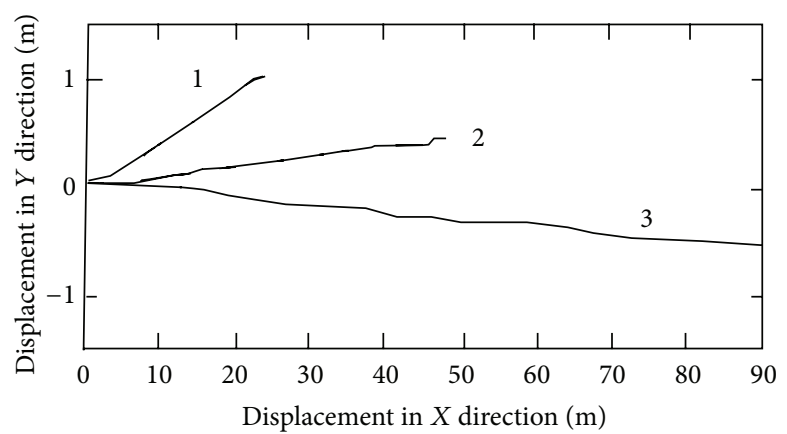

(f) The centroid trajectory

FIGURE 4: Characteristics of cars at different speeds on the low friction road. Curve 1: the speed is $14.4 \mathrm{~km} / \mathrm{h}$; Curve 2: the speed is $28.8 \mathrm{~km} / \mathrm{h}$; Curve 3: the speed is $54 \mathrm{~km} / \mathrm{h}$. 


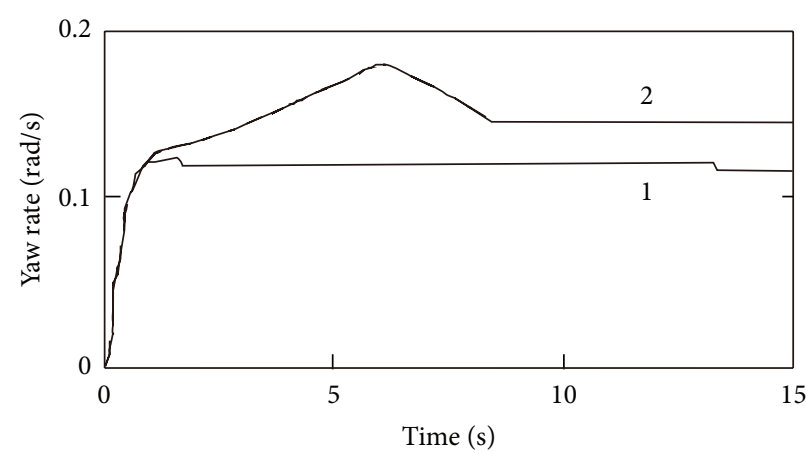

(a) The change of the yaw angular velocity

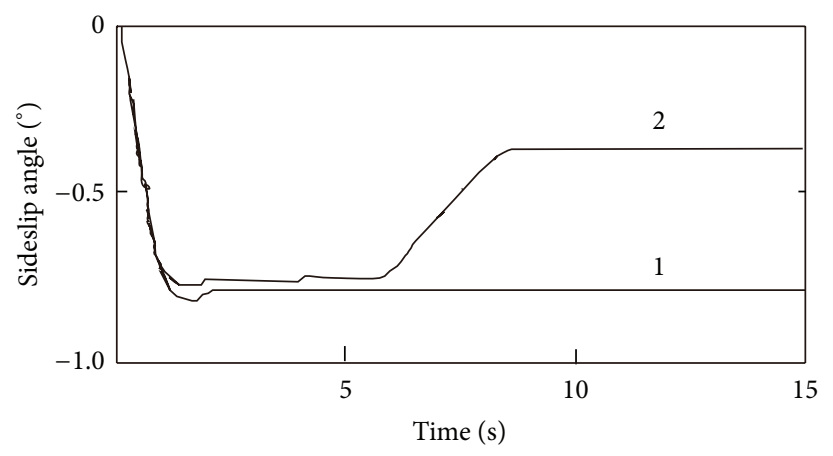

(c) The rear-wheel sideslip angles change

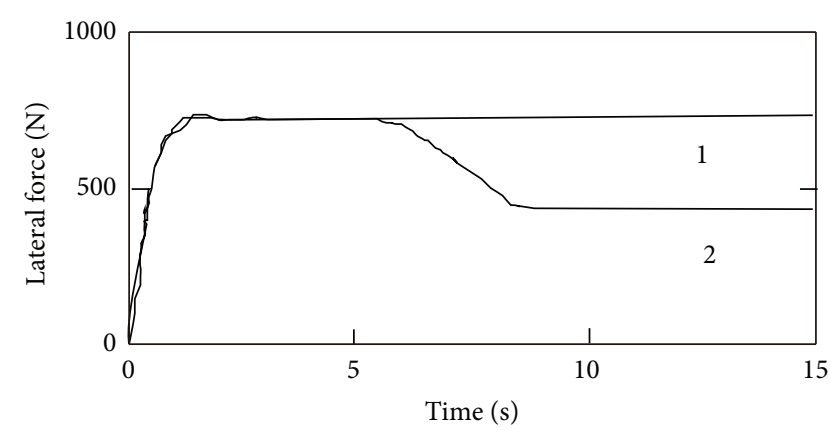

(e) Lateral force variation of the rear wheels

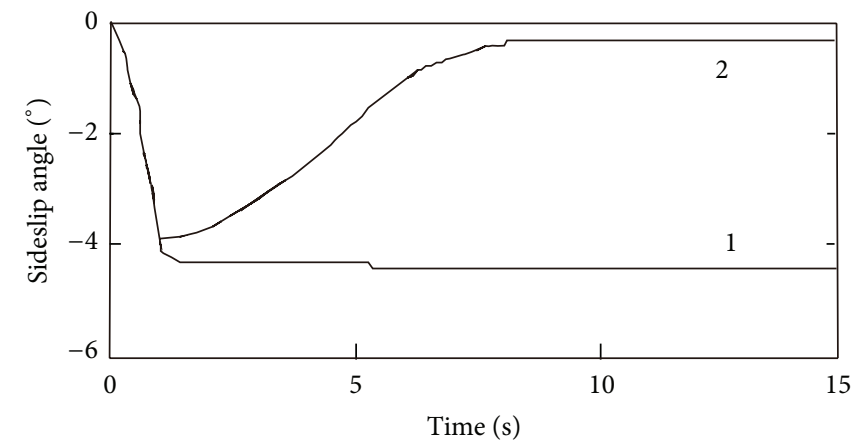

(b) The front-wheel sideslip angles change

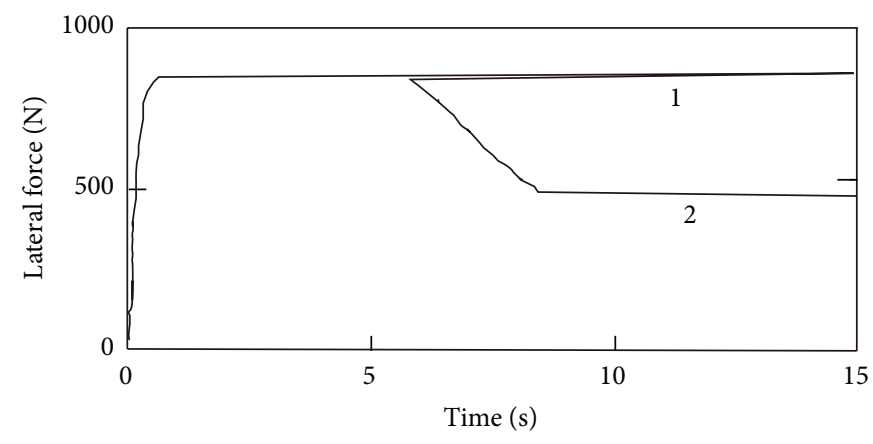

(d) Lateral force variation of the front wheels

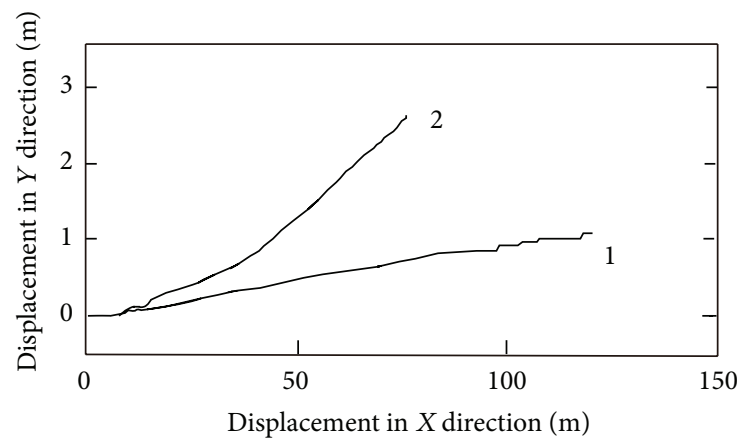

(f) The centroid trajectory

FIgURE 5: The characteristics of cars excessive sideslip on the low friction road and then slowing down. Curve 1: the speed is $28.8 \mathrm{~km} / \mathrm{h}$; Curve 2: the initial velocity is $28.8 \mathrm{~km} / \mathrm{h}$ and then is decreased to $14.4 \mathrm{~km} / \mathrm{h}$.

Define

$$
e=\Delta V_{N}-\Delta V_{A}
$$

$\theta_{\text {control }}$ stands for the actual control value of throttle opening; $\theta_{\text {input }}$ stands for input of the accelerator pedal opening by the diver; $\Delta V_{N}$ stands for the nominal value of wheel speed difference; and $\Delta V_{A}$ stands for the measured wheel speed difference.

The target control value of the throttle opening is the smaller one between the control value of the throttle opening and the accelerator pedal opening input by the driver:

$$
\theta_{\text {target }}=\min \left(\theta_{\text {input }}, \theta_{\text {control }}\right) \text {. }
$$

This formula guarantees that the final target control value of the throttle opening is no more than the command value input by the driver. The principles of vehicle dynamic stability control systems are illustrated in Figure 3.

\section{Cornering Stability Control Simulation and Test}

Figure 4 shows the curves produced by the simulation when the car is travelling at speeds of $14.4 \mathrm{~km} / \mathrm{h}, 28.8 \mathrm{~km} / \mathrm{h}$, and $54 \mathrm{~km} / \mathrm{h}$, respectively, on a road with a peak adhesion coefficient of 0.2 . This figure indicates that when the car is moving at a low speed, both the front wheels and the rear wheels are working in the linear zone. The yaw velocity is large and the movement locus of the center of mass is close to the ideal locus. As the vehicle's speed rises, however, the front wheels enter the nonlinear zone, resulting in reduced yaw velocity 


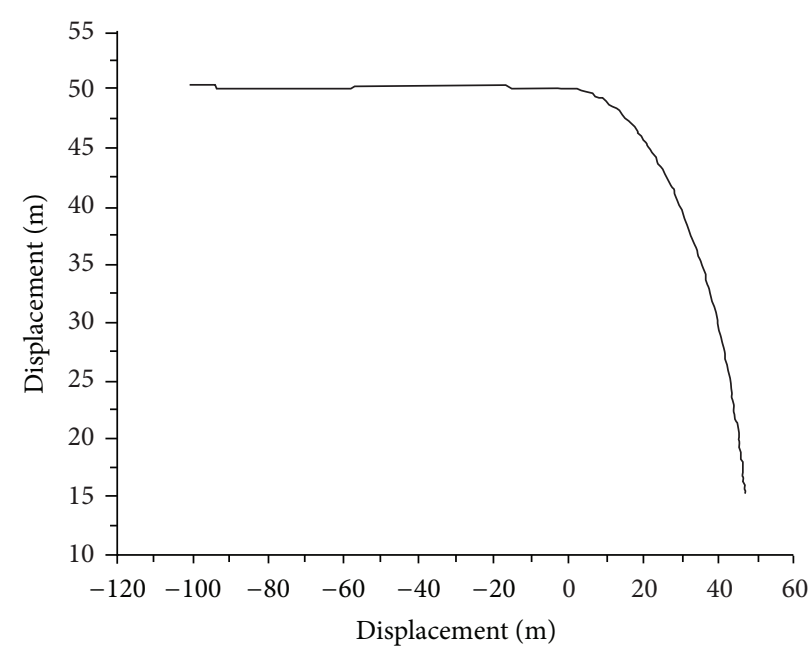

(a) Trajectory of the vehicle body

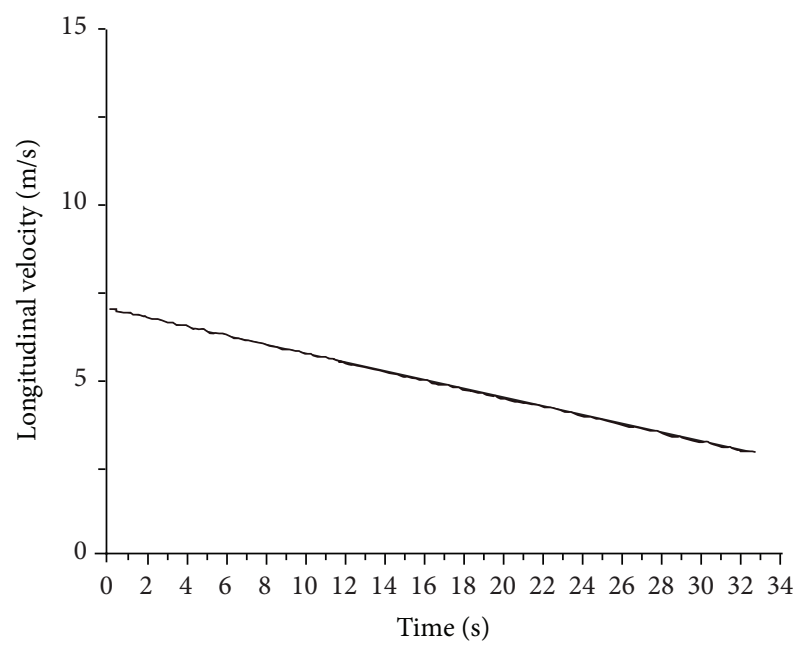

(c) Longitudinal velocity of centroid

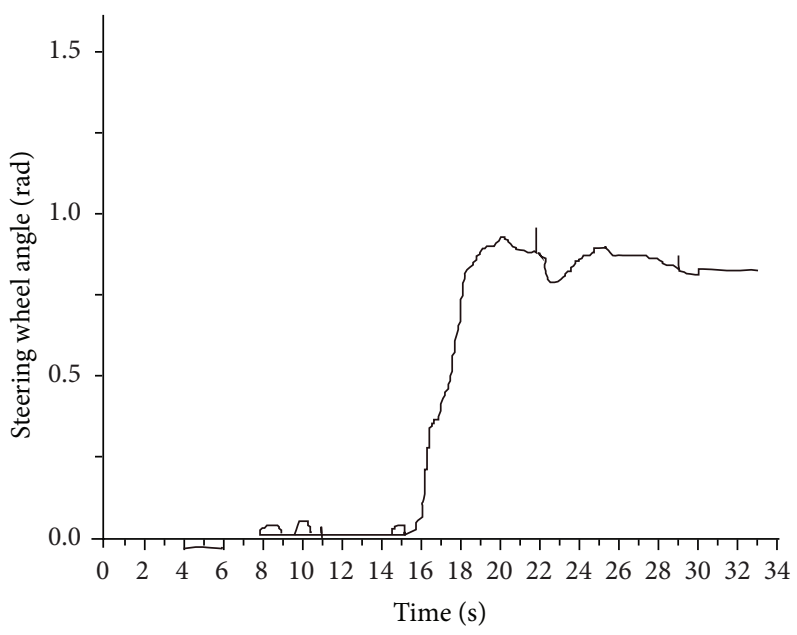

(b) Steering wheel angle

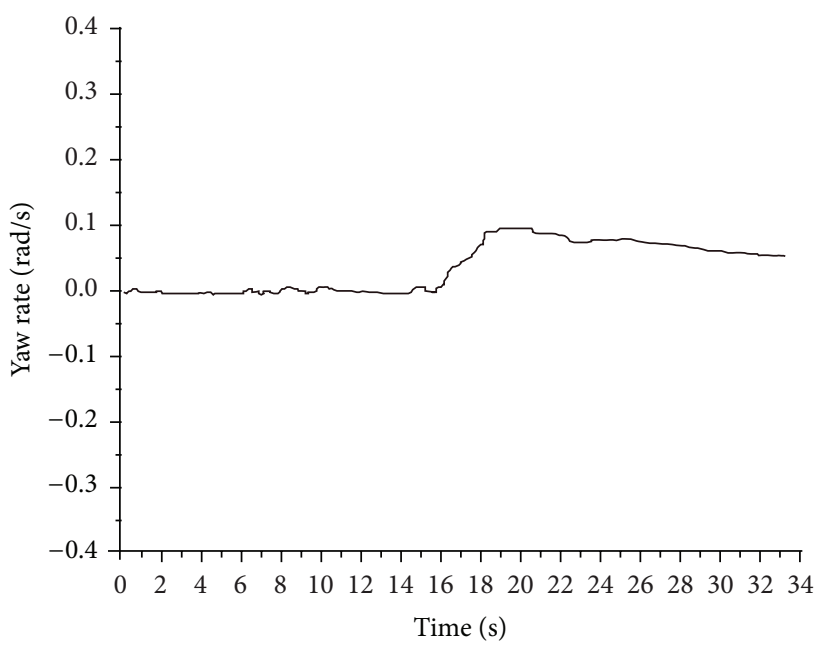

(d) Yaw rate of the vehicle

Figure 6: The vehicle cornering with excessive sideslip.

and a marked increase in the sideslip angle of the center of mass. During the simulation, the front-wheel cornering angle input is the same as in Figure 2(a).

Curve 1 in Figure 5 shows the cornering of a car on a road with a peak friction coefficient of 0.2 and at a speed of $28.8 \mathrm{~km} / \mathrm{h}$, with the front-wheel cornering angle input being the same as indicated in Figure 2(a). It can be clearly seen that the combination of high speed and low adhesion leads to a large sideslip angle, resulting in the tires entering the nonlinear zone. Curve 2 shows the cornering of a car under the same road adhesion and the front-wheel cornering angle conditions as Curve 1 , but the speed is decreasing from $28.8 \mathrm{~km} / \mathrm{h}$ to $14.4 \mathrm{~km} / \mathrm{h}$. As the speed of the car decreases, the sideslip angle of both the front and rear wheels decreases sharply, with the front wheels shifting from the nonlinear zone to the linear sideslip zone, greatly improving the lateral stability of the vehicle. Since lateral force has a linear relationship with the sideslip angle when the tires are working in the linear zone, this allows the driver to steer the vehicle more easily. Figure 5(a) shows the increased yaw angular velocity, while the sideslip can be seen to be distinctly reduced in Figures 5(b) and 5(c).

Figures 6 and 7 are the experimental results in the driving simulator in Jilin University, which is the largest one in China. As demonstrated in Figures 6 and 7, speed control can already enable a vehicle to corner smoothly under extremely low road adhesion conditions, but this does however result in big fluctuations in the steering wheel angle and yaw rate, which indicates that it is actually quite difficult to corner when the car is moving on a road with such a low adhesion, and hence destabilization can easily occur.

\section{Conclusion}

Though capable of greatly improving the cornering performance of vehicles travelling at high speeds and on roads with low adhesion, vehicle dynamic stability control systems are expensive. For cars already equipped with automatic transmission, dynamic stability control can actually be realized simply by installing two nondrive-wheel speed sensors to 


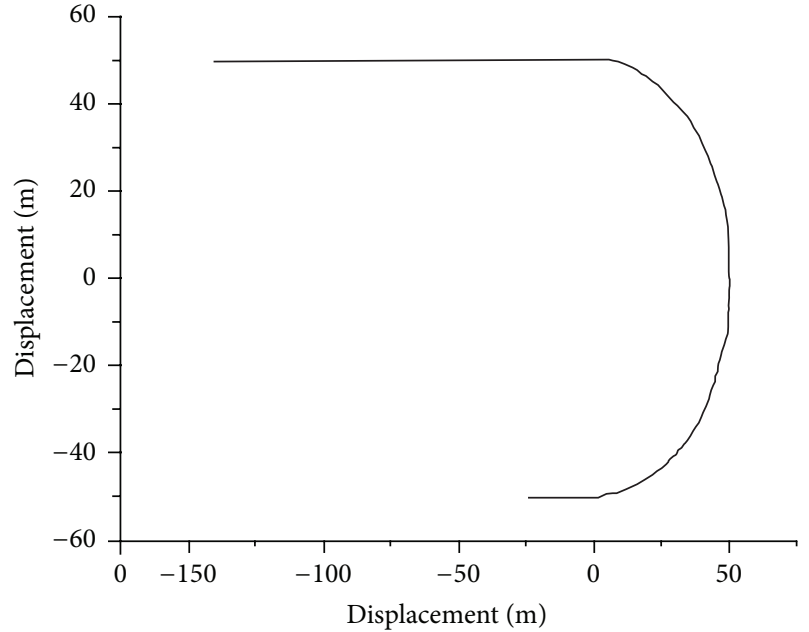

(a) Trajectory of the vehicle body

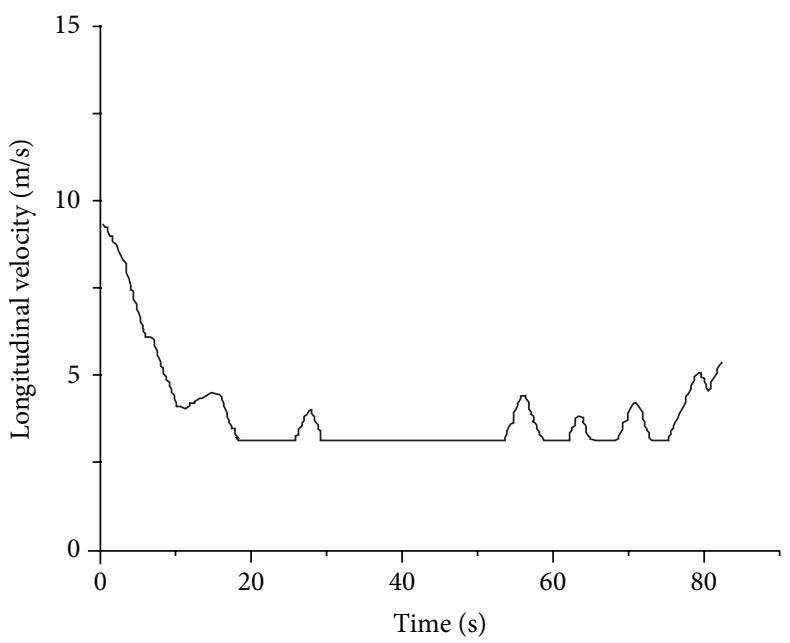

(c) Longitudinal velocity of centroid

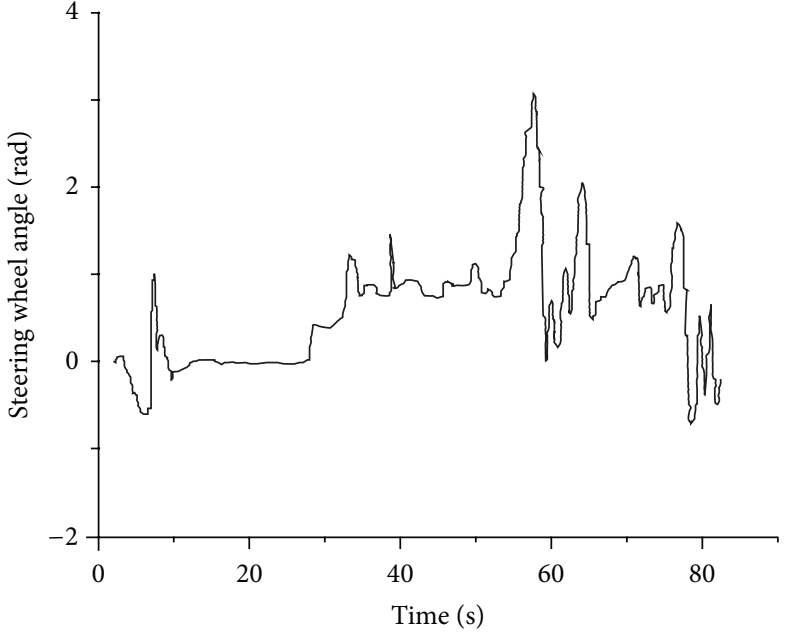

(b) Steering wheel angle

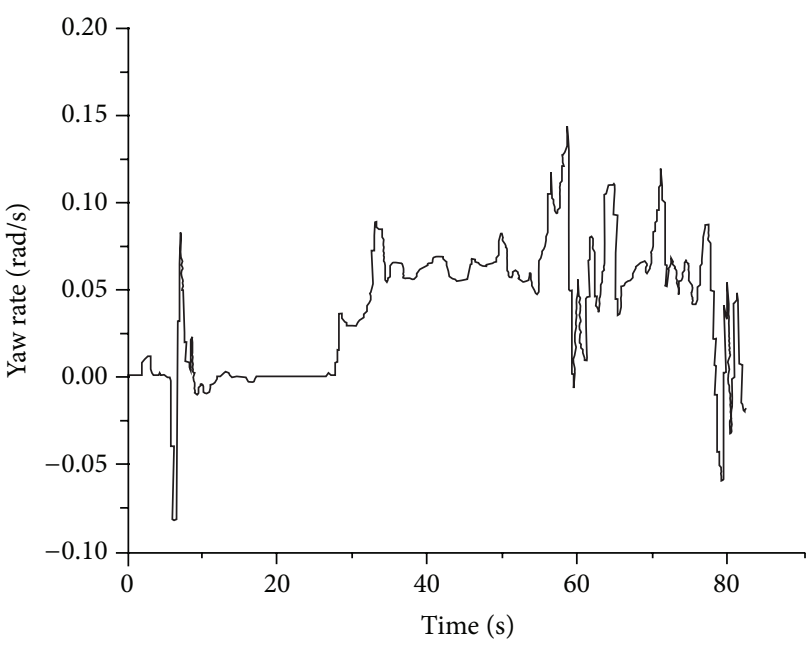

(d) Yaw rate of the vehicle

FIGURE 7: Vehicle concerning eliminating excessive sideslip.

the existing system. This paper designs a stability control system based on the wheel speed difference of nondrive wheels, which can be compared with the speed predicted by the linear 2-degree-of-freedom vehicle model in order to estimate the yaw rate and sideslip angular speed of a vehicle. Simulation tests are conducted on excessive sideslip, nonsideslip, and the elimination of excessive sideslip through speed control for vehicles moving at different speeds on roads of low adhesion, with tests of excessive sideslip and nonsideslip cornering being performed in a driving simulator. The system developed in this paper is quite inexpensive and could greatly improve the active safety of AT vehicles on the crooked roads and hence has great application potential.

\section{Conflict of Interests}

The authors declare that there is no conflict of interests regarding the publishing of this paper.

\section{Acknowledgment}

This work was supported by the National Natural Science Foundation of China (Grant no. 51375053).

\section{References}

[1] S. Di Cairano, H. E. Tseng, D. Bernardini, and A. Bemporad, "Vehicle yaw stability control by coordinated active front steering and differential braking in the tire sideslip angles domain," IEEE Transactions on Control Systems Technology, vol. 21, no. 4, pp. 1236-1248, 2013.

[2] G. R. Frumusanu, I. C. Constantin, V. Marinescu, and A. Epureanu, "Development of a stability intelligent control system for turning," International Journal of Advanced Manufacturing Technology, vol. 64, no. 5-8, pp. 643-657, 2013.

[3] W. Cho, J. Choi, C. Kim, S. Choi, and K. Yi, "Unified chassis control for the improvement of agility, maneuverability, and lateral stability," IEEE Transactions on Vehicular Technology, vol. 61, no. 3, pp. 1008-1020, 2012. 
[4] S. Oh, H. Fujimoto, Y. Hori, and K. Nam, "Robust yaw stability control for electric vehicles based on active front steering control through a steer-by-wire system," International Journal of Automotive Technology, vol. 13, no. 7, pp. 1169-1176, 2012.

[5] L. Wang, N. Zhang, and H. Du, "Experimental investigation of a hydraulically interconnected suspension in vehicle dynamics and stability control," SAE International Journal of Passenger Cars-Mechanical Systems, vol. 5, no. 2, pp. 759-768, 2012.

[6] J. Tjoønnäs and T. A. Johansen, "Stabilization of automotive vehicles using active steering and adaptive brake control allocation," IEEE Transactions on Control Systems Technology, vol. 18, no. 3, pp. 545-558, 2010.

[7] A. Carvalho, G. Palmieri, H. E. Tseng, L. Glielmo, and F. Borrelli, "Robust vehicle stability control with an uncertain driver model," in Proceedings of the 12th European Control Conference (ECC '13), pp. 440-445, IEEE, July 2013.

[8] H. Du, N. Zhang, and F. Naghdy, "Velocity-dependent robust control for improving vehicle lateral dynamics," Transportation Research Part C: Emerging Technologies, vol. 19, no. 3, pp. 454468, 2011.

[9] S. Li and L. He, "Co-simulation study of vehicle ESP system based on ADAMS and MATLAB," Journal of Software, vol. 6, no. 5, pp. 866-872, 2011.

[10] B. Li and F. Yu, "Design of a vehicle lateral stability control system via a fuzzy logic control approach," Proceedings of the Institution of Mechanical Engineers. Part D. Journal of Automobile Engineering, vol. 224, no. 3, pp. 313-326, 2010.

[11] H. Koto, T. Kato, C. Nitta, K. Suzuki, and T. Yamada, "Enhancement of yaw and roll stability for trucks by estimating payload conditions," SAE International Journal of Commercial Vehicles, vol. 5, no. 1, pp. 94-100, 2012.

[12] K. Bayar, J. Wang, and G. Rizzoni, "Development of a vehicle stability control strategy for a hybrid electric vehicle equipped with axle motors," Proceedings of the Institution of Mechanical Engineers Part D: Journal of Automobile Engineering, vol. 226, no. 6, pp. 795-814, 2012.

[13] M. Jinlai, W. Bofu, C. Jie, and Z. Zhongliang, "Comparisons of vehicle stability controls based on 4WS, Brake, Brake-FAS and IMC techniques," Vehicle System Dynamics, vol. 50, no. 7, pp. 1053-1084, 2012.

[14] H. Zhu, L. Li, M. Jin, H. Li, and J. Song, "Real-time yaw rate prediction based on a non-linear model and feedback compensation for vehicle dynamics control," Proceedings of the Institution of Mechanical Engineers, Part D: Journal of Automobile Engineering, vol. 227, no. 10, pp. 1431-1445, 2013.

[15] H. Zhou and Z. Liu, "Vehicle yaw stability-control system design based on sliding mode and backstepping control approach," IEEE Transactions on Vehicular Technology, vol. 59, no. 7, pp. 3674-3678, 2010.

[16] C. Novara, F. Ruiz, and M. Milanese, "Direct identification of optimal SM-LPV filters and application to vehicle yaw rate estimation," IEEE Transactions on Control Systems Technology, vol. 19, no. 1, pp. 5-17, 2011.

[17] X. Wu, M. Xu, and L. Wang, "Differential speed steering control for four-wheel independent driving electric vehicle," in Proceedings of the IEEE 22nd International Symposium on Industrial Electronics (ISIE '13), May 2013.

[18] K. Mahmud and L. Tao, "Vehicle speed control through fuzzy logic," in Proceedings of the IEEE Global High Tech Congress on Electronics (GHTCE '13), pp. 30-35, November 2013.
[19] J. Wang, Q. Wang, L. Jin, and C. Song, "Independent wheel torque control of 4WD electric vehicle for differential drive assisted steering," Mechatronics, vol. 21, no. 1, pp. 63-76, 2011.

[20] H. Leffler, "Consideration of lateral and longitudinal vehicle stability by function enhanced brake and stability control system," SAE Technical Paper 940832, 1994. 


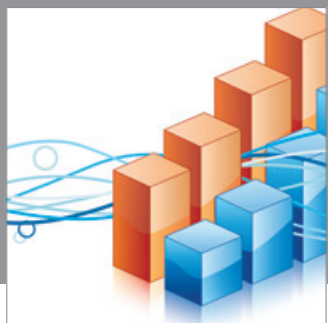

Advances in

Operations Research

mansans

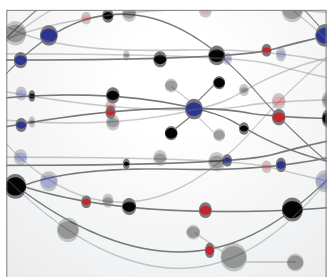

The Scientific World Journal
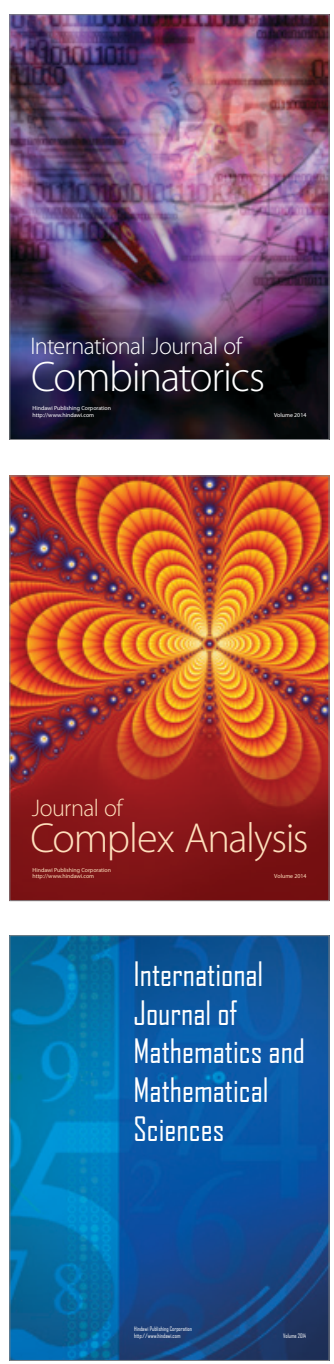
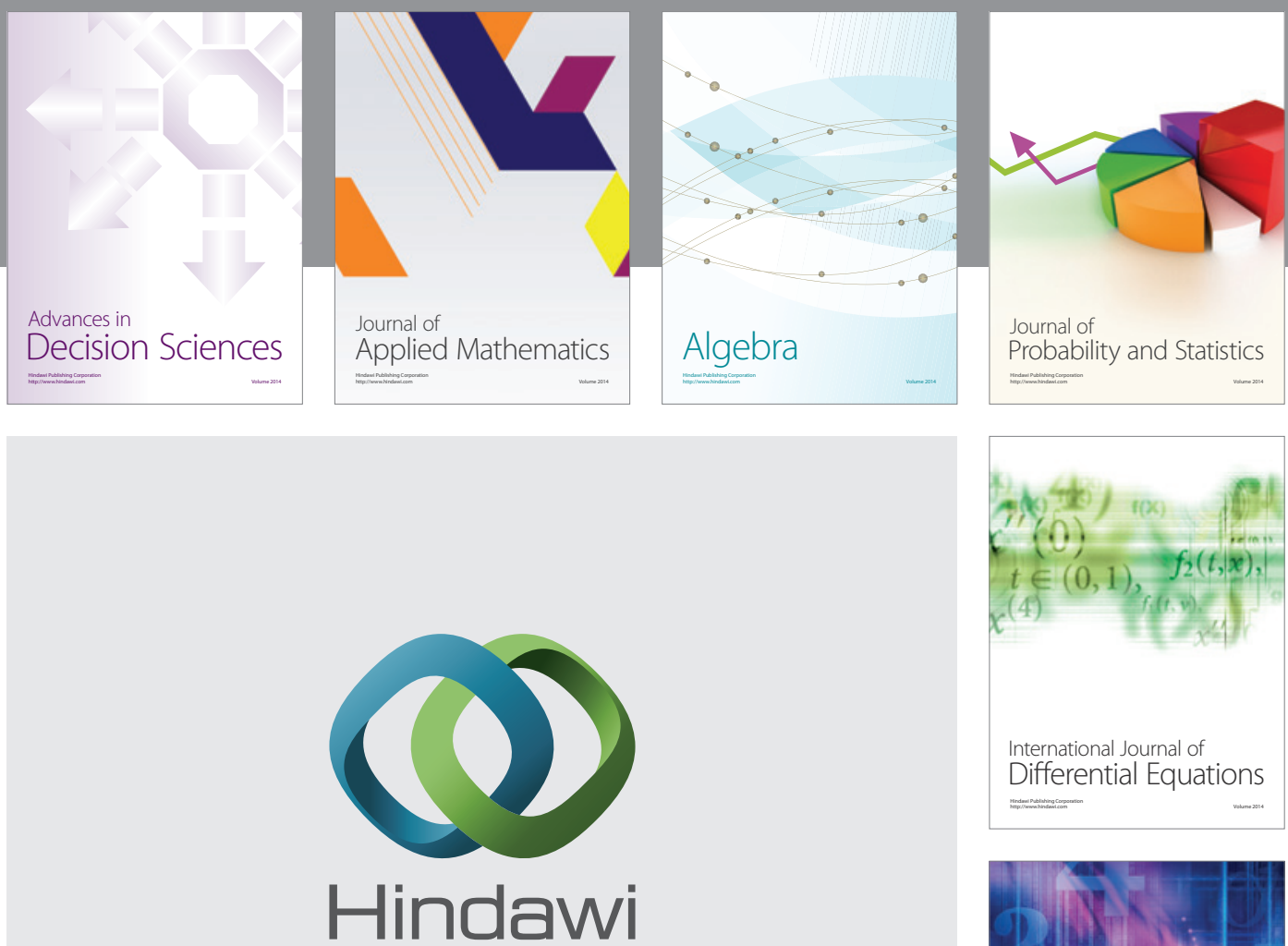

Submit your manuscripts at http://www.hindawi.com
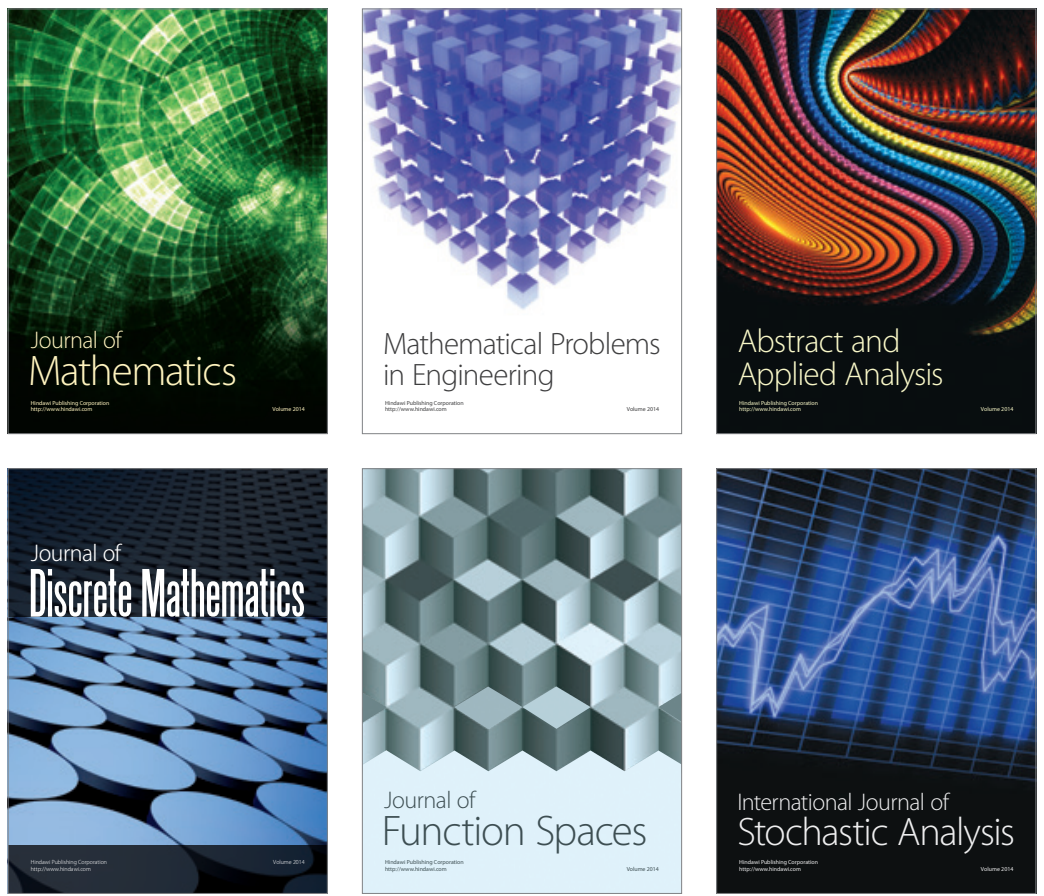

Journal of

Function Spaces

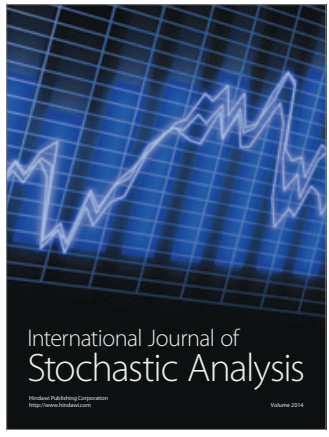

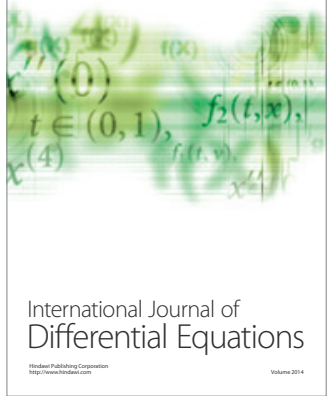
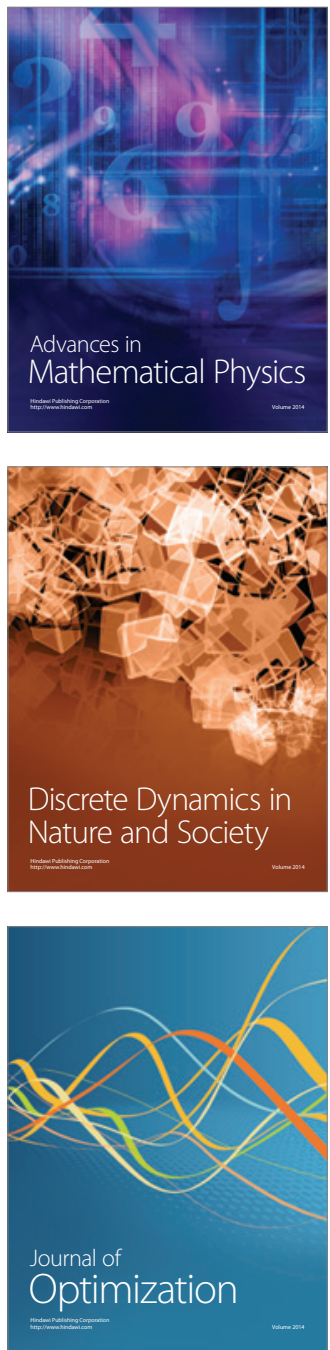